Case Report

\title{
Atypical Lipomatous Tumor/Well-Differentiated Liposarcoma with Intramuscular Lipoma-Like Component of the Thigh
}

\author{
Chairat Burusapat $\mathbb{D}^{1}{ }^{1}$ Nuttadon Wongprakob $\mathbb{D}^{1}{ }^{1}$ Nutthapong Wanichjaroen $\mathbb{D}^{1}{ }^{1}$ \\ Chatchai Pruksapong $\left(\mathbb{D},{ }^{1}\right.$ and Kantang Satayasoontorn $\mathbb{1}^{2}$ \\ ${ }^{1}$ Division of Plastic and Reconstructive Surgery, Department of Surgery, Phramongkutklao Hospital and Phramongkutklao College \\ of Medicine, Bangkok, Thailand \\ ${ }^{2}$ Department of Pathology and Laboratory Medicine, Phramongkutklao Hospital, Bangkok, Thailand \\ Correspondence should be addressed to Chairat Burusapat; pataranat@hotmail.com
}

Received 19 May 2020; Revised 1 December 2020; Accepted 5 December 2020; Published 14 December 2020

Academic Editor: Yoshiharu Kawaguchi

Copyright (C) 2020 Chairat Burusapat et al. This is an open access article distributed under the Creative Commons Attribution License, which permits unrestricted use, distribution, and reproduction in any medium, provided the original work is properly cited.

\begin{abstract}
Atypical lipomatous tumor/well-differentiated liposarcoma (ALT/WDLPS) is a locally aggressive mesenchymal neoplasm composed either entirely or partly of an adipocytic proliferation showing at least focal nuclear atypia in both adipocytes and stromal cells. ALT most frequently occurs in deep soft tissue of proximal extremities (thigh and buttock) and usually presents as a deep-seated, painless mass that can slowly attain a very large size, which is one of the most common sarcomas of extremity. In the patients who presented with deep-seated tumor, distinction between intramuscular lipoma and well-differentiated liposarcoma is important due to the differences in treatment and prognosis. However, atypical well-differentiated liposarcoma with intramuscular lipoma-like component of the thigh is extremely rare. Moreover, the infiltrative growth pattern or intramuscular component may lead to a misinterpretation as intramuscular lipoma on a small biopsy. We present an unusual case of a female who presented with symptomatic mass at the thigh which has rarely been reported in English literature as an infiltrative intramuscular lipoma-like growth pattern of well-differentiated liposarcoma. Therefore, preoperative diagnosis is necessary, and correlation with imaging studies is required when one encounters with a large deep-seated mass. Atypical lipomatous tumor or well-differentiated liposarcoma should be kept in mind in the patient who presents with abnormal thigh mass.
\end{abstract}

\section{Introduction}

Atypical lipomatous tumor/well-differentiated liposarcoma (ALT/WDLPS) is a locally aggressive mesenchymal neoplasm composed either entirely or partly of an adipocytic proliferation showing at least focal nuclear atypia in both adipocytes and stromal cells. "Atypical lipomatous tumor" and "well-differentiated liposarcoma" are synonyms describing lesions that are morphologically and genetically identical. Amplification of $M D M 2$ and/or CDK4 is almost always present. ALT/WDLPS represents the largest subgroup of adipocytic malignancies, accounting for approximately $40-45 \%$ of all liposarcomas. These lesions occur predominantly in middle-aged adults, with peak incidence between the fourth and fifth decades of life. ALT most frequently occurs in deep soft tissue of proximal extremities (thigh and buttock) and usually presents as a deep-seated, painless mass that can slowly attain a very large size [1]. In the patients who presented with deep-seated tumor, distinction of intramuscular lipoma and ALT/WDLPS is important due to the different treatments and prognosis. While ALT/WDLPS usually consists of a large, well-circumscribed, lobulated mass, some cases have partial replacement of surrounding muscle tissue with entrapped striated muscle fibers resembling intramuscular lipoma. Generally, the presence of infiltrative margins and intermingled muscle fibers in intramuscular lipoma are a characteristic and indicates a benign lesion rather than malignancy on magnetic resonance imaging $[2,3]$. This 


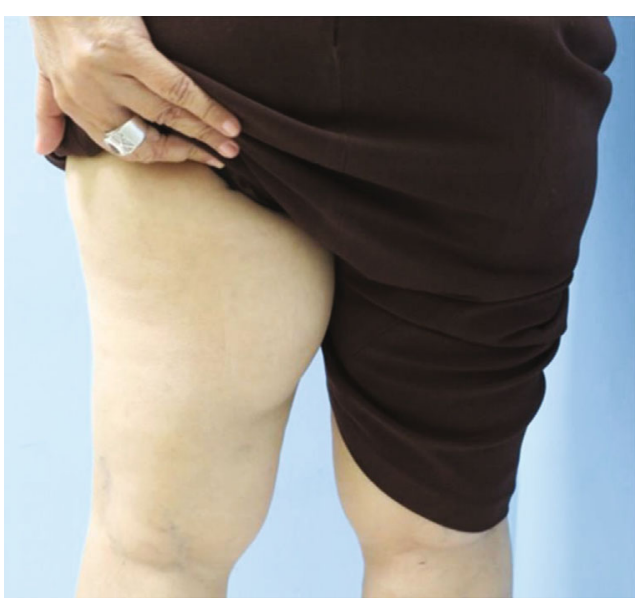

(a)

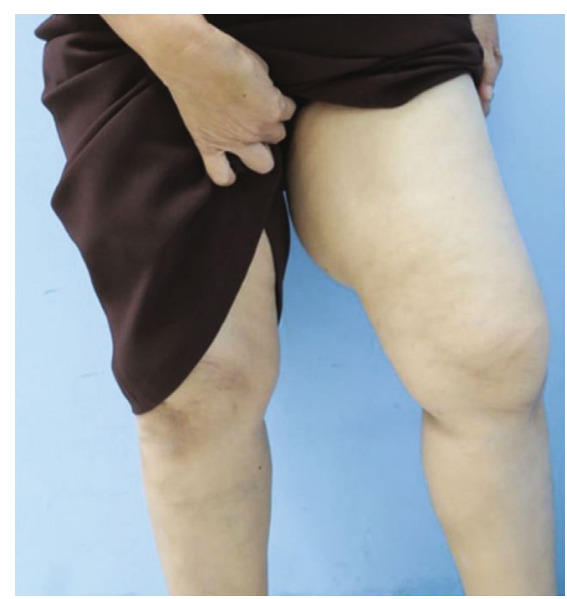

(b)

FIgURE 1: Demonstrated mass at left thigh: (a) posterior view, (b) front view.

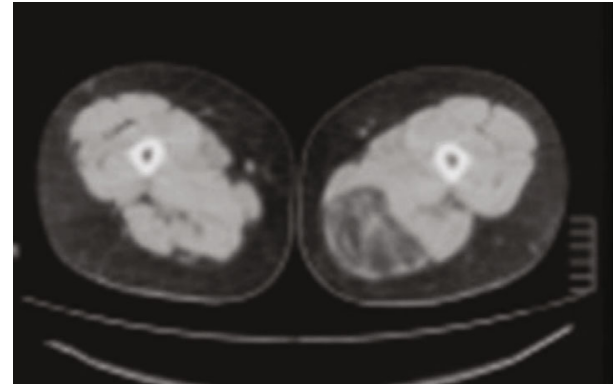

(a)

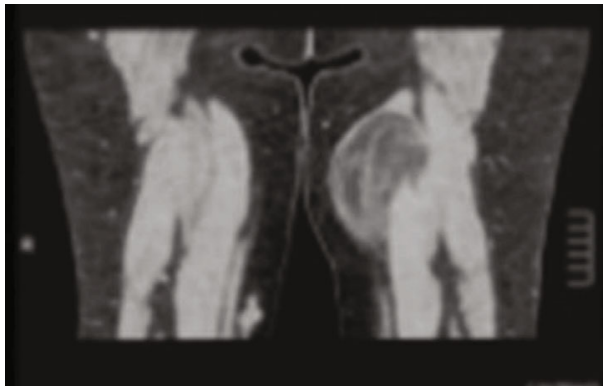

(b)

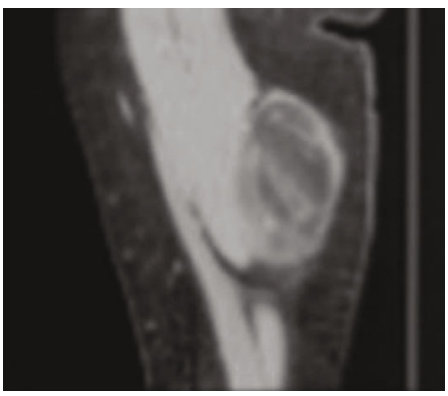

(c)

Figure 2: Computed tomography (CT) scan demonstrated $11 \times 8 \times 7 \mathrm{~cm}$, in vertical x transverse $\mathrm{x}$ AP diameter of well-defined, fatty lesion with thick streak fibrous tissue at the right posteromedial of thigh: (a) axial view, (b) coronal view, and (c) sagittal view.

component of ALT/WDLPS may cause confusion with intramuscular lipoma on histologic examination and lead to a misdiagnosis. Careful sampling of these tumors is mandatory because portions of an intramuscular atypical lipomatous neoplasm may be indistinguishable from intramuscular lipoma [4].

Here, we present a rare case of ALT/WDLPS with obvious infiltrative intramuscular component which can be confused with intramuscular lipoma.

\section{Case Report}

A 61-year-old Thai woman presented with a nontender mass at the left posterior thigh rapidly growing for 6 months. She had no any underlying or previous surgery. She had pain when she walked and slightly limited her left leg function during motion. On physical examination, there was a firm nonpulsatile mass over left medial thigh measuring $\sim 10 \mathrm{x} 6$ $\mathrm{cm}$ in diameter (Figure 1). There was no notable grossly skin involvement, and the mass appears not fixed to bony structure. No sensory deficit is identified. The left inguinal lymph node cannot be palpated.

The computed tomography (CT) scan demonstrated an intramuscular $11 \times 8 \times 7 \mathrm{~cm}$, in vertical xtransverse $\mathrm{AP}$ diameter of well-defined, fatty lesion with thin streak fibrous tissue at the left posteromedial of the thigh (Figure 2). Fine needle biopsy (FNA) suggested lipoma but liposarcoma cannot be excluded. Then, the core needle biopsy was performed 


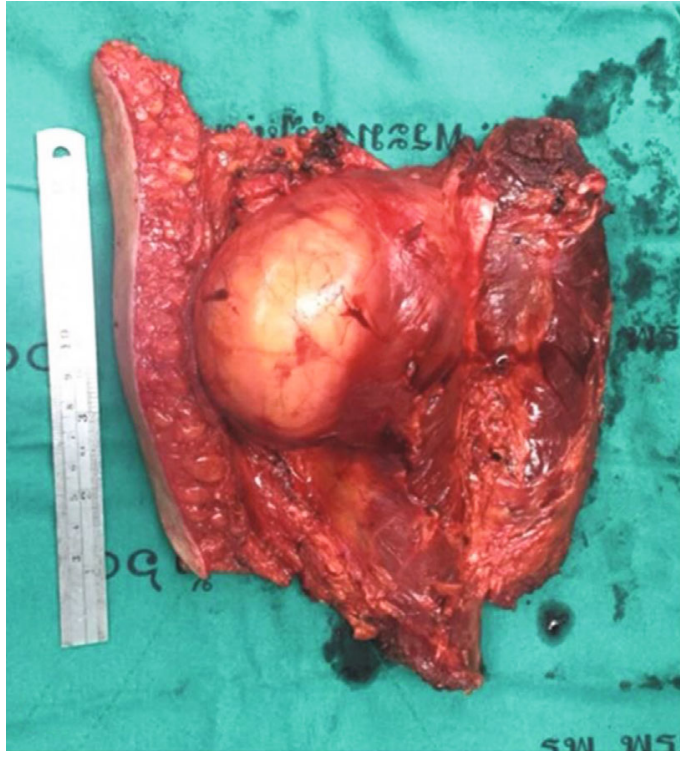

(a)

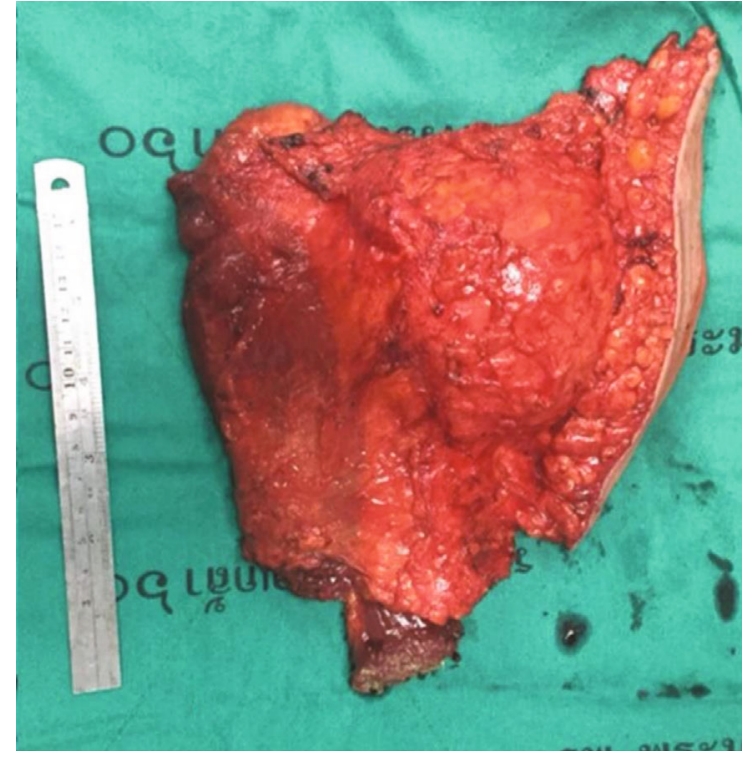

(b)

Figure 3: Complete surgical removal of tumor.

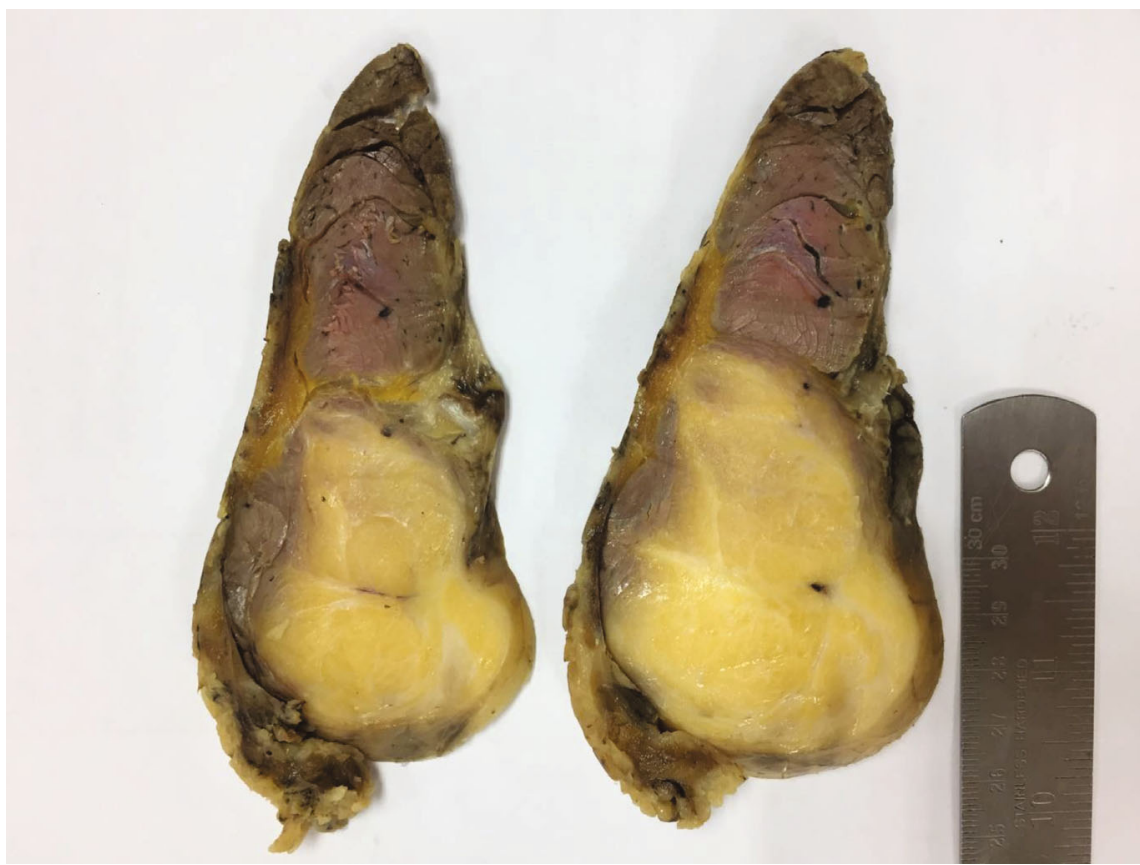

Figure 4: The gross specimen showed cut surfaces of the mass revealing pale yellow, greasy, lobulated mass with ill-defined, infiltrative edge to the surrounding muscle on one side.

and showed well-differentiated liposarcoma. Metastasis workups were unremarkable. The operation was performed on supine position under general anesthesia. Vertical incision was performed. The tumor was confined in the semimembranosus and semitendinosus muscle. Complete en bloc tumor resection with total removal of semimembranosus and semitendinosus muscles was successful (Figure 3). The gross specimen consisted an intramuscular mass, measuring $20 \times 17 \times 6 \mathrm{~cm}$ with yellow, lobulated, rubbery, rather well-circumscribed cut surfaces. The mass is generally well delineated from the surrounding tissues by a thin white translucent, glistening membrane. Serial sections of the mass revealed focal gradual replacement of the muscle tissue by fat into the intermuscular connective tissue spaces (Figure 4). The other areas showed yellow smooth rubbery cut surface. Microscopic examination showed an adipocytic neoplasm. The tumor was mainly composed of small and large adipocytes with interspersed fibrous septa that contain large, irregular, hyperchromatic nuclei. Small nucleoli presented in some of the large cells. There were scattered atypical cells 


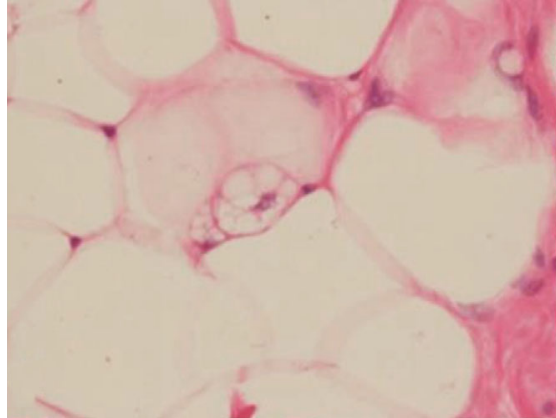

(a)

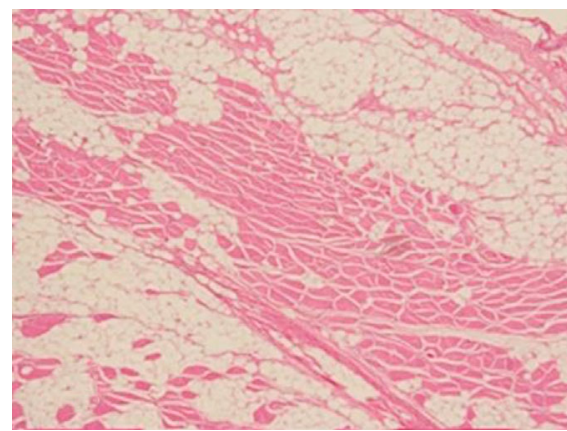

(c)

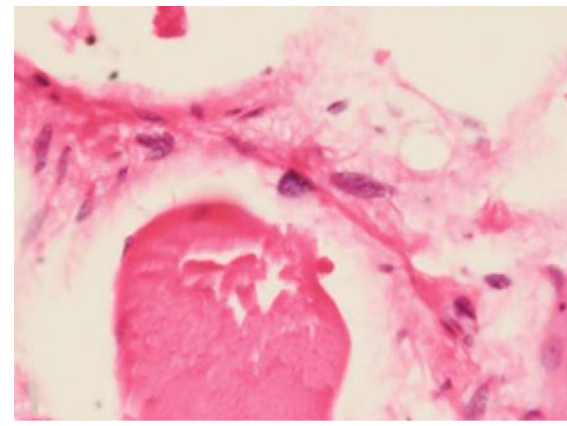

(e)

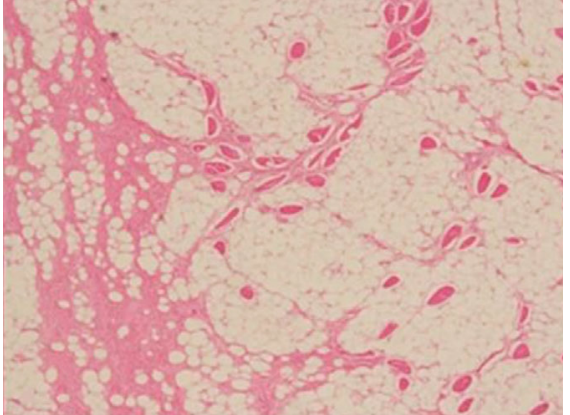

(b)

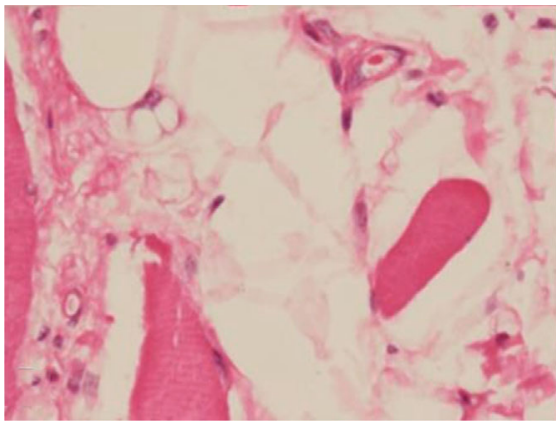

(d)

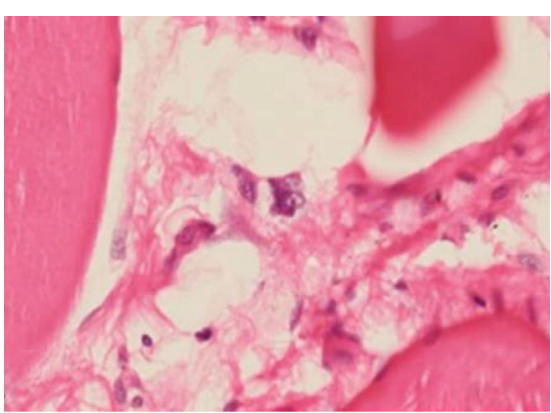

(f)

Figure 5: Microscopic examination. (a) Histological revealed characteristic multivacuolated lipoblasts. (b, c) An infiltrative, intramuscular component is composed of adipocytic lesion encasing skeletal muscle fibers. (d-f) Multivacuolated lipoblast and hyperchromatic stromal cells found in between the muscle fibers.

containing large hyperchromatic nuclei in collagenous background. Intramuscular component resembling intramuscular lipoma at periphery of the mass was also presented characterized by adipocytes diffusely infiltrating muscle. The entrapped muscle fibers usually show few changes other than various degrees of muscular atrophy. There were also few lipoblasts and stromal cells with atypical nuclei in fibrous septa between muscle fibers as seen in ALT/WDLPS present. No necrosis and no high-grade sarcomatous component were seen (Figure 5). The final pathologic diagnosis was well-differentiated liposarcoma with intramuscular lipomalike component.

\section{Discussion}

Well-differentiated liposarcoma is the most common histologic subtype of liposarcoma, accounting for $40 \%$ to $45 \%$ of all liposarcoma therefore representing the larger subgroup of adipocytic malignancies. Well-differentiated liposarcoma is subdivided in the adipocytic (lipoma-like), sclerosing, inflammatory, and spindle cell subtypes, of which the adipocytic-like and sclerosing-type are common [5].

Adipocytic well-differentiated liposarcoma is composed of a relatively mature adipocytic proliferation, featuring cell size variation as well as at least focal nuclear atypia. Varying number (from many to none) of lipoblasts may be found [5]. However, an infiltrative growth pattern or intramuscular component can be rarely seen in this reported case. The infiltrative growth pattern or intramuscular component may lead to a misinterpretation as intramuscular lipoma on a small biopsy. Therefore, correlation with imaging studies is required when one encounter with a large deep-seated mass.

Distinguishing between intramuscular lipoma and welldifferentiated liposarcoma can be difficult based on histologic analysis alone. Because of differences in treatment, prognosis, 
and long-term follow-up, it is important to preoperatively distinguish simple lipoma from well-differentiated liposarcoma [6]. Correct classification is important, because aggressive local disease recurrence occurs more frequently in patients with well-differentiated liposarcoma than in patients with intramuscular lipoma [7].

Computed tomography (CT) and magnetic resonance imaging (MRI) have been used for preoperative identify between intramuscular lipoma and well-differentiated liposarcoma. CT and MRI revealed fatty lesions containing streaky structures in benign lesions. In welldifferentiated liposarcoma, thick streaks represented entrapped muscle fibers, and thin streaks represented fibrous tissue or neoplastic spindle cell proliferation. Furthermore, CT in well-differentiated liposarcoma revealed foci of hazy amorphous density, representing spindle cell proliferation [8]. As for the well-differentiated liposarcoma, thick septa and nodular or patchy nonadipose components were present more frequently in deep lesions than in subcutaneous lesions. The septa in welldifferentiated liposarcoma enhanced more strongly than in benign lipoma. The septa showed no enhancement relative to muscle in benign lipoma, whereas the septa showed moderate or marked enhancement in all welldifferentiated liposarcoma [9].

Moreover, MRI revealed significantly enhanced signal in well-differentiated liposarcoma in a background of multiple well-differentiated benign fatty masses by showing the increased vascularity in the septa of well-differentiated liposarcoma. Although such signal enhancement can be seen in some types of benign lipomatous tumors with increased blood vessels, this technique is helpful in selection of biopsy site, especially in a clinical setting of multiple fatty masses [10].

When the data for solitary lipoma were compared to soft-tissue sarcoma, it was found that patient age and duration of symptoms were of minor value in the clinical differential diagnosis. However, if a tumor was larger than $5 \mathrm{~cm}$, irrespective of depth and location, located in the thigh, irrespective of depth and size, or deep, irrespective of location and size, it was more likely to be a sarcoma [10].

Patients with extremity tumors had a significantly better prognosis than those with retroperitoneal or scrotal tumors. Extremity tumors treated by wide local excision recurred in only $11 \%$ of cases, whereas $60 \%$ of those treated by marginal or simple excision recurred [11]. Although, metastases and deaths due to tumor had never been reported, Evans et al. reported $17 \%$ suffered inoperable recurrence and $10 \%$ died as a result of the neoplasm [12].

Intramuscular lipomas were smaller than welldifferentiated liposarcoma, but there was significant overlap between the 2 groups. Four percent of patients with intramuscular lipoma and $27 \%$ of patients with welldifferentiated liposarcoma developed local disease recurrence [7]. Disease recurrence did not correlate with patient age at diagnosis, gender, tumor size, and tumor location.

A well-differentiated liposarcoma of any type has no potential for metastasis unless they undergo dedifferentiation
[5]. Occasionally, the terms atypical lipoma, atypical intramuscular lipoma, and well-differentiated liposarcoma made the plastic surgeon confused. Well-differentiated liposarcoma and atypical lipoma should be considered synonyms, and their use should therefore be determined by the degree of reciprocal comprehension between the surgeon and the pathologist to prevent either inadequate or excessive treatment.

Generally, when soft tissue tumor exists throughout the body in large sizes, if deeply located and infiltrating, a diagnosis of a soft tissue sarcoma must be considered [13]. Positive resection margins were associated with reduced local recurrence-free survival [14].

A review of the literature showed only two cases of retroperitoneal liposarcoma associated with multiple subcutaneous lipomas, two cases of liposarcoma involving an extremity associated with multiple subcutaneous lipomas, and one case of intramuscular liposarcoma associated with multiple intramuscular lipomas [15].

\section{Conclusion}

Atypical well-differentiated liposarcoma with intramuscular lipoma-like component of the thigh is extremely rare. The infiltrative growth pattern or intramuscular component may lead to a misinterpretation as intramuscular lipoma on a small biopsy. Therefore, preoperative diagnosis is necessary and correlation with imaging studies is required when one encounter with a large deep-seated mass. Atypical lipomatous tumor or well-differentiated liposarcoma should be kept in mind in the patient who presents with abnormal thigh mass.

\section{Ethical Approval}

For case reports, our institute was exempted to take ethical approval.

\section{Consent}

Informed consent for the publication of this work has been given by the patient.

\section{Conflicts of Interest}

The authors declare that they have no conflicts of interest.

\section{References}

[1] M. Sbaraglia, A. P. Dei Tos, and F. Pedeutour, "Atypical lipomatous tumour/well-differentiated liposarcoma," in Soft tissue and bone tumors/edited by WHO Classification of Tumours Editorial Board, pp. 36-38, IARCLyon, Lyon, 5th edition, 2020.

[2] I. Chernev and S. Tingey, "Thenar intramuscular lipoma: a case report," Journal of Medical Cases, vol. 4, pp. 676-678, 2013.

[3] K. Matsumoto, S. Hukuda, M. Ishizawa, T. Chano, and H. Okabe, "MRI findings in intramuscular lipomas," Skeletal Radiology, vol. 28, pp. 145-152, 1999. 
[4] J. R. Goldblum, A. L. Folpe, and S. W. Weiss, "Benign lipomatous tumors," in Enzinger and Weiss's Soft Tissue Tumors, pp. 501-503, Elsevier, Philadelphia, PA, USA, 7th edition, 2020.

[5] L. Laurino, A. Furlanetto, E. Orvieto, and A. P. Dei Tos, "Welldifferentiated liposarcoma (atypical lipomatous tumors)," Seminars in Diagnostic Pathology, vol. 18, pp. 258-262, 2001.

[6] C. M. Gaskin and C. A. Helms, "Lipomas, lipoma variants, and well-differentiated liposarcomas (atypical lipomas): results of MRI evaluations of 126 consecutive fatty masses," AJR. American Journal of Roentgenology, vol. 182, pp. 733-739, 2004.

[7] M. D. Bassett, S. M. Schuetze, C. Disteche et al., "Deep-seated, well differentiated lipomatous tumors of the chest wall and extremities: the role of cytogenetics in classification and prognostication," Cancer, vol. 103, pp. 409-416, 2005.

[8] J. Nishida, T. Morita, A. Ogose et al., "Imaging characteristics of deep-seated lipomatous tumors: intramuscular lipoma, intermuscular lipoma, and lipoma-like liposarcoma," Journal of Orthopaedic Science, vol. 12, pp. 533-541, 2007.

[9] T. Ohguri, T. Aoki, M. Hisaoka et al., "Differential diagnosis of benign peripheral lipoma from well-differentiated liposarcoma on MR imaging: is comparison of margins and internal characteristics useful?," AJR. American Journal of Roentgenology, vol. 180, pp. 1689-1694, 2003.

[10] Y. J. Yang, T. A. Damron, H. Cohen, and L. Hojnowski, "Distinction of well-differentiated liposarcoma from lipoma in two patients with multiple well-differentiated fatty masses," Skeletal Radiology, vol. 30, pp. 584-589, 2001.

[11] D. R. Lucas, A. G. Nascimento, B. K. Sanjay, and M. G. Rock, "Well-differentiated liposarcoma. The Mayo Clinic experience with 58 cases," American Journal of Clinical Pathology, vol. 102, pp. 677-683, 1994.

[12] H. L. Evans, E. H. Soule, and R. K. Winkelmann, "Atypical lipoma, atypical intramuscular lipoma, and well differentiated retroperitoneal liposarcoma: a reappraisal of 30 cases formerly classified as well differentiated liposarcoma," Cancer, vol. 43, pp. 574-584, 1979.

[13] M. Hoshi, S. Matsumoto, J. Manabe et al., "Surgery for dedifferentiated liposarcoma, presenting two radiologically and pathologically distinctive patterns," Japanese Journal of Clinical Oncology, vol. 36, pp. 462-467, 2006.

[14] D. A. Kooby, C. R. Antonescu, M. F. Brennan, and S. Singer, "Atypical lipomatous tumor/well-differentiated liposarcoma of the extremity and trunk wall: importance of histological subtype with treatment recommendations," Annals of Surgical Oncology, vol. 11, pp. 78-84, 2004.

[15] K. Matsumoto, S. Hukuda, M. Ishizawa, M. Egawa, and H. Okabe, "Liposarcoma associated with multiple intramuscular lipomas. A case report," Clinical Orthopaedics and Related Research, vol. 373, pp. 202-207, 2000. 